Tonghua Zhu, Xiuhua Liu, Jianguo Qin, Qingyuan Hu, Jiamin Ning

Institute of Nuclear Physics and Chemistry, China Academy of Engineering Physics, Mianyang 621999, China

Studies on the luminescence and scintillation properties of 9,10-diphenylanthracene Crystals grown by two methods 

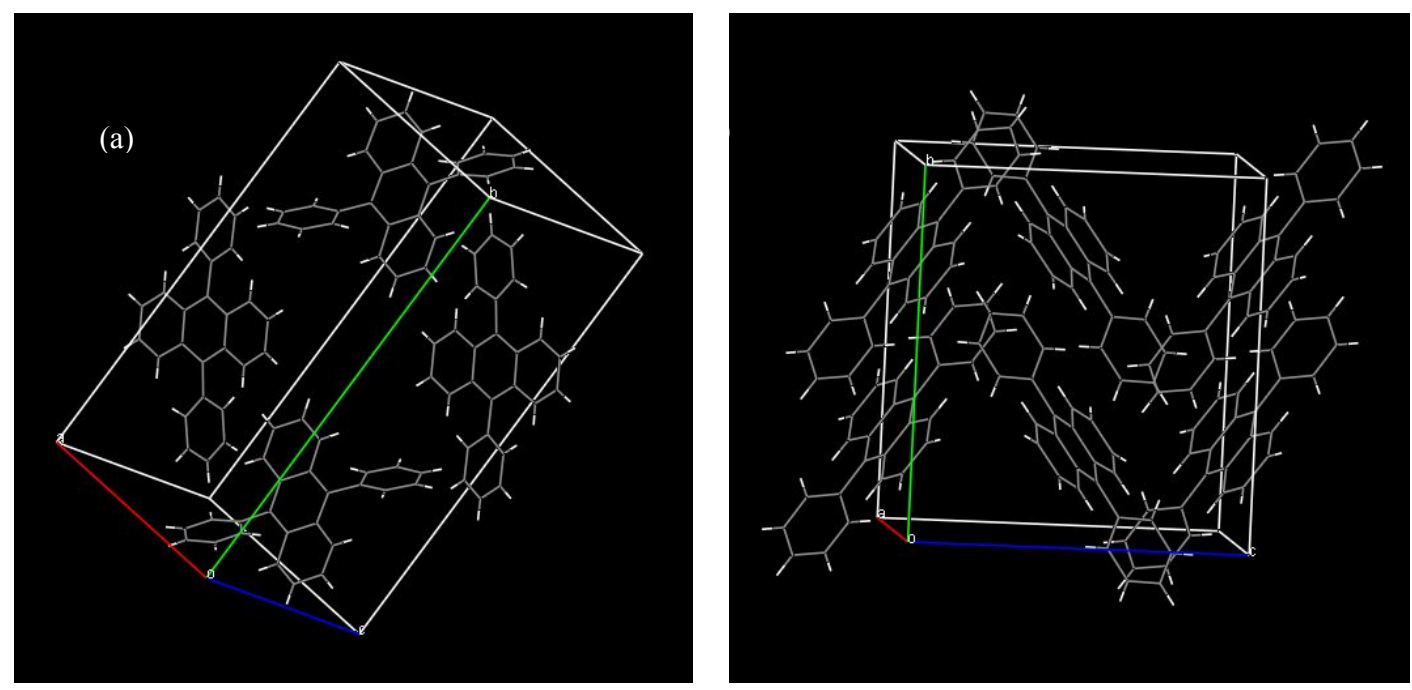

Figure 1 Unit cells of (a) DPA-Melt and (b) DPA-Solution.

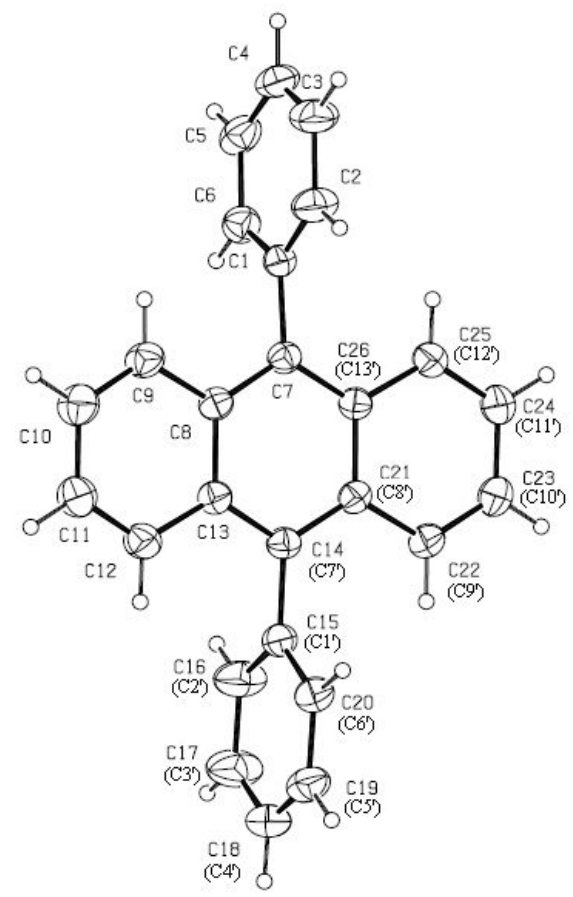

Figure 2 DPA molecule. 
Figure 3 Crystallographic data of DPA-M and DPA-S.

\begin{tabular}{|c|c|c|}
\hline Crystal & DPA-M & DPA-S \\
\hline Empirical formula & $\mathrm{C}_{26} \mathrm{H}_{18}$ & $\mathrm{C}_{13} \mathrm{H}_{9}$ \\
\hline Temperature/K & 293(2) & 293.15 \\
\hline Formula weight & 330.40 & 165.20 \\
\hline Crystal system & Monoclinic & Monoclinic \\
\hline Space group & $\mathrm{P} 2{ }_{1} / \mathrm{n}$ & $\mathrm{C} 2 / \mathrm{c}$ \\
\hline $\mathrm{a} / \AA$ & $9.2004(4)$ & $10.6842(7)$ \\
\hline $\mathrm{b} / \AA$ & $21.0836(8)$ & $13.5461(8)$ \\
\hline $\mathrm{c} / \AA$ & $10.0367(4)$ & $12.2430(8)$ \\
\hline$\alpha /^{\circ}$ & 90 & 90 \\
\hline$\beta /{ }^{\circ}$ & $111.446(5)$ & $90.615(6)$ \\
\hline$\gamma /{ }^{\circ}$ & 90 & 90 \\
\hline Volume $/ \AA^{3}$ & $1812.10(14)$ & 1771.82(19) \\
\hline Z & 4 & 8 \\
\hline$\rho_{\text {calc }} \mathrm{g} / \mathrm{cm}^{3}$ & 1.211 & 1.239 \\
\hline$\mu / \mathrm{mm}^{-1}$ & 0.068 & 0.070 \\
\hline $\mathrm{F}(000)$ & 696.0 & 696.0 \\
\hline Crystal size $/ \mathrm{mm}^{3}$ & $0.2 \times 0.08 \times 0.05$ & $0.4 \times 0.35 \times 0.25$ \\
\hline Radiation & $\operatorname{MoK} \alpha(\lambda=0.71073)$ & $\operatorname{MoK} \alpha(\lambda=0.71073)$ \\
\hline $2 \Theta$ range for data collection ${ }^{\circ}$ & 7.256 to 52.736 & 5.864 to 52.734 \\
\hline Index ranges & $-11 \leq \mathrm{h} \leq 8,-26 \leq \mathrm{k} \leq 20,-12 \leq 1 \leq 12$ & $-13 \leq \mathrm{h} \leq 11,-16 \leq \mathrm{k} \leq 16,-8 \leq 1 \leq 15$ \\
\hline Reflections collected & 11425 & 3856 \\
\hline Independent reflections & $3699\left[\mathrm{R}_{\mathrm{int}}=0.0278, \mathrm{R}_{\text {sigma }}=0.0332\right]$ & $1823[$ Rint $=0.0222$, Rsigma $=0.0408]$ \\
\hline Data/restraints/parameters & $3699 / 0 / 236$ & $1823 / 0 / 118$ \\
\hline
\end{tabular}


Final R indexes $[\mathrm{I}>=2 \sigma(\mathrm{I})]$

Final R indexes [all data]
$\mathrm{R}_{1}=0.0523, \mathrm{wR}_{2}=0.1286$

$\mathrm{R}_{1}=0.0802, \mathrm{wR}_{2}=0.1470$
$\mathrm{R}_{1}=0.0533, \mathrm{wR}_{2}=0.1141$

$\mathrm{R} 1=0.0772, \mathrm{wR} 2=0.1320$ 


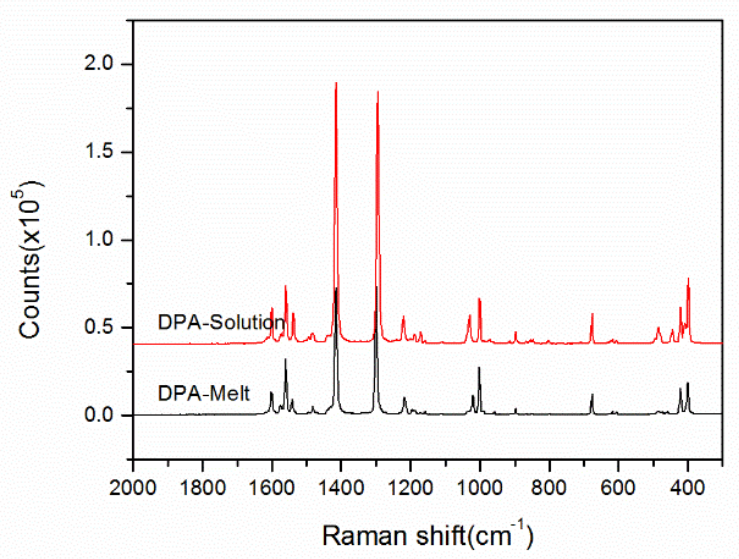

Figure 4 Raman spectra of the DPA crystals.

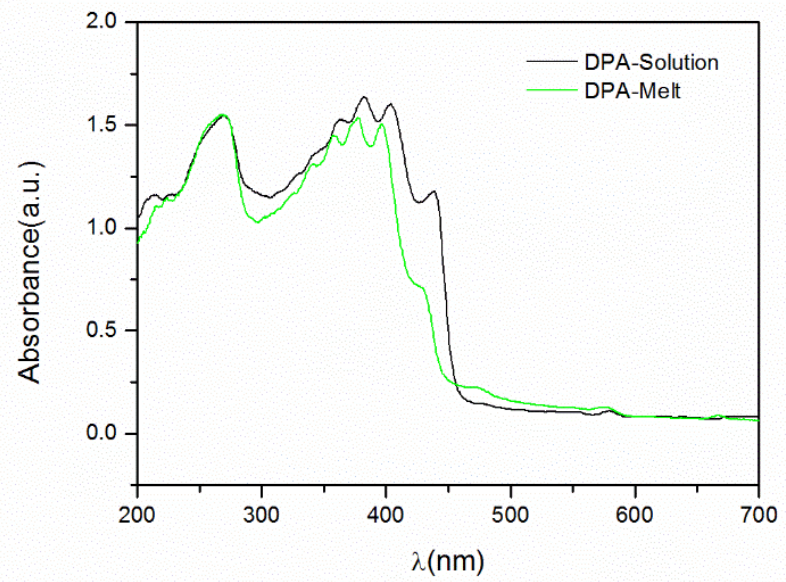

Figure 5 UV-Vis absorption spectra of the DPA crystals.

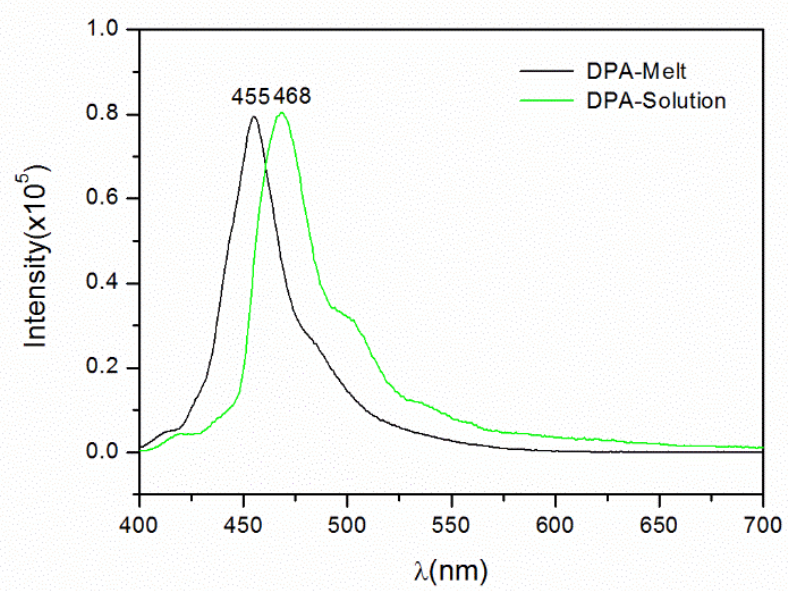

Figure 6 Fluorescence emission spectra of the DPA crystals. 

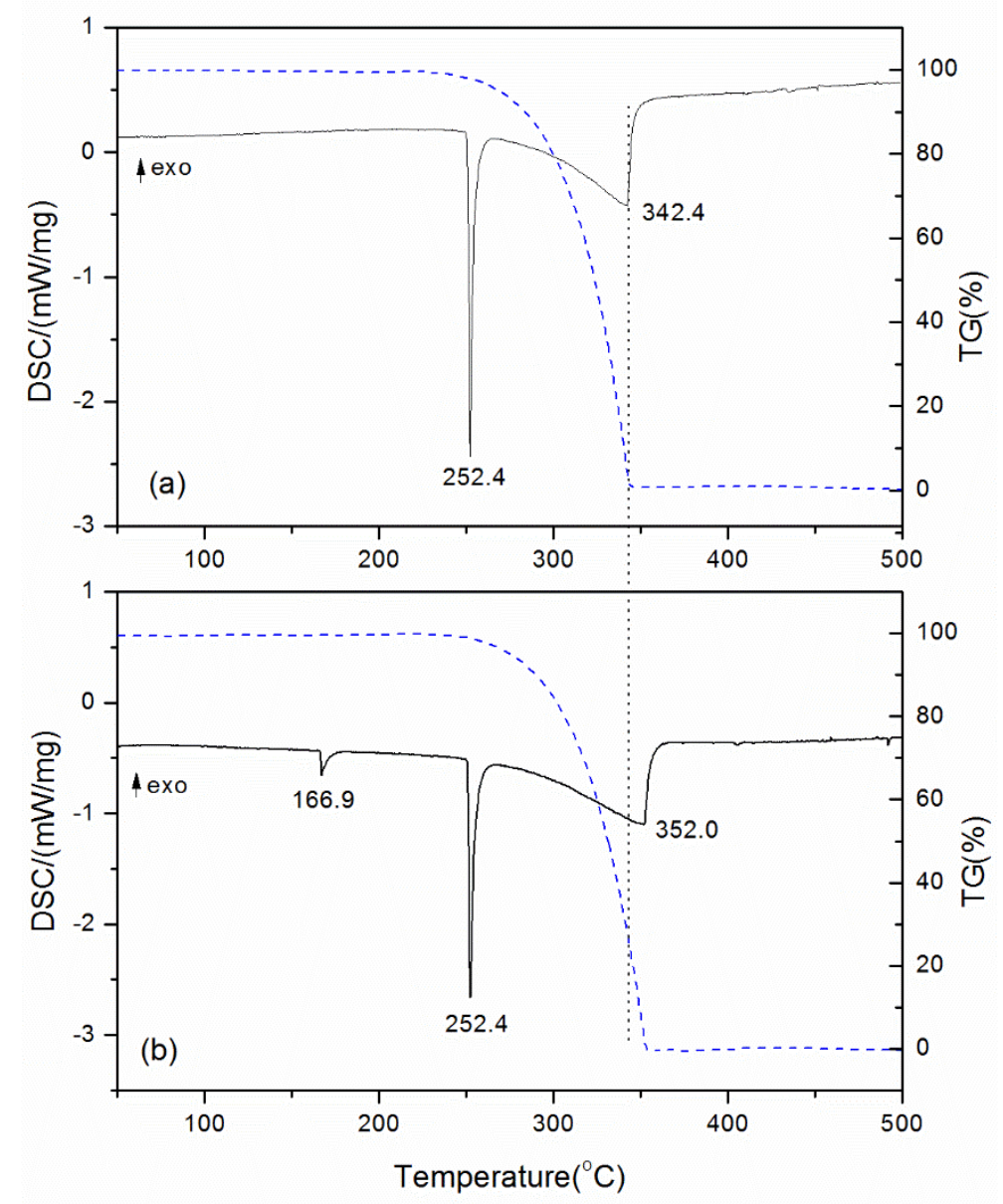

Figure 7 TG-DSC curves of (a) DPA-Melt and (b) DPA-Solution crystals. 
CheckCIF Report 
DPA-Solution

No syntax errors found.

Please wait while processing .... $\underline{\text { CIF dictionary }}$

Interpreting this report

\section{Datablock: 180122_s2_1xh_2}

Bond precision: $\quad \mathrm{C}-\mathrm{C}=0.0021 \mathrm{~A} \quad$ Wavelength $=0.71073$

Ce11: $\quad \mathrm{a}=10.6842(7) \quad \mathrm{b}=13.5461(8) \quad \mathrm{c}=12.2430(8)$

$$
\text { alpha }=90 \quad \text { beta }=90.615 \text { (6) gamma }=90
$$

Temperature: $293 \mathrm{~K}$

$$
\text { Calculated Reported }
$$

Volume

1771. $82(19)$

1771. $82(19)$

Space group

C $2 / \mathrm{c}$

C $12 /$ c 1

Hall group

$-C 2 \mathrm{yc}$

$-\mathrm{C} 2 \mathrm{yc}$

Moiety formula

C26 H18

C26 H18

Sum formula

C26 H18

C26 H18

$\mathrm{Mr}$

330.40

330. 40

Dx, g cm-3

1. 239

1. 239

Z

4

4 


$\begin{array}{lll}\text { Mu }(\mathrm{mm}-1) & 0.070 & 0.070 \\ \text { F000 } & 696.0 & 696.0 \\ \text { F000' } & 696.26 & 13,16,15 \\ \text { h, k, 1max } & 13,16,15 & 1823 \\ \text { Nref } & 1824 & 0.937,1.000 \\ \text { Tmin, Tmax } & 0.972,0.983 & \\ \text { Tmin' } & 0.972 & \end{array}$

Correction method= \# Reported T Limits: $T \min =0.937 \operatorname{Tmax}=1.000$

AbsCorr $=$ MULTI-SCAN

Data completeness $=0.999 \quad$ Theta $(\max )=26.367$

$\mathrm{R}($ reflections $)=0.0533(1305) \quad$ wR2 (reflections $)=0.1320(1823)$

$S=1.073 \quad \quad$ Npar $=118$

The following ALERTS were generated. Each ALERT has the format test-name_ALERT_alert-type_alert-level.

Click on the hyperlinks for more details of the test.

\section{QAlert level A}

EXPT005 ALERT 1 A _expt1_crystal_description is missing

Crystal habit description.

The following tests will not be performed. 
CRYSR_01

- Alert level C

PLAT918 ALERT 3 C Reflection(s) with I (obs) much Smaller I(calc) .

1 Check

PLAT978 ALERT 2 C Number C-C Bonds with Positive Residual Density.

0 Info

Alert leve1 G

PLAT199 ALERT 1 G Reported_cel1_measurement_temperature ..... (K)

293 Check

PLAT200_ALERT 1 G Reported_diffrn_ambient_temperature ..... (K)

293 Check

PLAT910 ALERT 3 G Missing \# of FCF Reflection(s) Below Theta(Min).

1 Note

1 ALERT level $\mathrm{A}=$ Most likely a serious problem - resolve or explain

0 ALERT level $\mathrm{B}=\mathrm{A}$ potentially serious problem, consider carefully

2 ALERT leve1 $\mathrm{C}=$ Check. Ensure it is not caused by an omission or oversight

3 ALERT leve1 $\mathrm{G}=$ General information/check it is not something unexpected

3 ALERT type 1 CIF construction/syntax error, inconsistent or missing data

1 ALERT type 2 Indicator that the structure model may be wrong or deficient

2 ALERT type 3 Indicator that the structure quality may be low

0 ALERT type 4 Improvement, methodology, query or suggestion 
0 ALERT type 5 Informative message, check

It is advisable to attempt to resolve as many as possible of the alerts in all categories. Often the minor alerts point to easily fixed oversights, errors and omissions in your CIF or refinement strategy, so attention to these fine details can be worthwhile. In order to resolve some of the more serious problems it may be necessary to carry out additional measurements or structure refinements. However, the purpose of your study may justify the reported deviations and the more serious of these should normally be commented upon in the discussion or experimental section of a paper or in the "special_details" fields of the CIF. checkCIF was carefully designed to identify outliers and unusual parameters, but every test has its limitations and alerts that are not important in a particular case may appear. Conversely, the absence of alerts does not guarantee there are no aspects of the results needing attention. It is up to the individual to critically assess their own results and, if necessary, seek expert advice.

\section{Publication of your CIF in IUCr journals}

A basic structural check has been run on your CIF. These basic checks will be run on all CIFs submitted for publication in IUCr journals (Acta Crystallographica, Journal of Applied Crystallography, Journal of Synchrotron Radiation); however, if you intend to submit to Acta Crystallographica Section C or E or IUCrData, you should make sure that full publication checks are run on the final version of your CIF prior to submission.

\section{Publication of your CIF in other journals}

Please refer to the Notes for Authors of the relevant journal for any special instructions relating to CIF submission.

\section{PLATON version of $03 / 05 / 2019$; check. def file version of $29 / 04 / 2019$}


Datablock 180122_s2_1xh_2 - ellipsoid plot

Download CIF editor (publCIF) from the IUCr

Download CIF editor (enCIFer) from the CCDC

Test a new CIF entry 
DPA-Melt

No syntax errors found.

$\underline{\text { CIF dictionary }}$

Please wait while processing ....

Interpreting this report

\section{Datablock: 20151125-hfp-1uo-1}

\begin{tabular}{|c|c|c|c|}
\hline Bond & $\mathrm{C}-\mathrm{C}$ & $0.0031 \mathrm{~A}$ & Wavelength $=0.71073$ \\
\hline \multirow[t]{2}{*}{ Cel1: } & $\mathrm{a}=9.2004(4)$ & $\mathrm{b}=21.0836(8)$ & $c=10.0367(4)$ \\
\hline & alpha $=90$ & beta $=111.446$ & gamma $=90$ \\
\hline
\end{tabular}

Temperature: $293 \mathrm{~K}$

Calculated Reported

Volume $\quad 1812.10(14) \quad 1812.10(14)$

Space group

P 21/n

P $121 /$ n 1

Ha11 group

$-P 2 y n$

$-P 2 y n$

Moiety formula

C26 H18

C26 H18

Sum formula

C26 H18

C26 H18

$\mathrm{Mr}$

330. 40

330. 40

Dx, g cm-3

1. 211

1. 211

Z

4 


$\begin{array}{lll}\text { Mu }(\mathrm{mm}-1) & 0.068 & 0.068 \\ \text { F000 } & 696.0 & 696.0 \\ \text { F000' } & 696.26 & 11,26,12 \\ \text { h, k, 1max } & 11,26,12 & 3699 \\ \text { Nref } & 3707 & 0.815,1.000 \\ \text { Tmin, Tmax } & 0.993,0.997 & \\ \text { Tmin’ } & 0.986 & \end{array}$

Correction method $=$ \# Reported T Limits: Tmin=0.815 Tmax=1. 000

AbsCorr $=$ MULTI-SCAN

Data completeness $=0.998 \quad$ Theta $(\max )=26.368$

$\mathrm{R}($ reflections $)=0.0523(2507) \quad$ wR2 $($ reflections $)=0.1470(3699)$

$\mathrm{S}=1.068 \quad$ Npar $=236$

The following ALERTS were generated. Each ALERT has the format test-name_ALERT_alert-type_alert-leve1.

Click on the hyperlinks for more details of the test.

\section{QAlert level A}

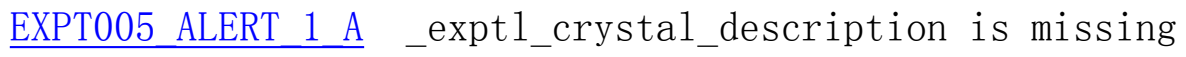

Crystal habit description.

The following tests will not be performed. 
CRYSR_01

\section{- Alert level C}

PLAT241 ALERT 2 C High 'MainMol' Ueq as Compared to Neighbors of

C17 Check

PLAT906 ALERT 3 C Large $\mathrm{K}$ Value in the Analysis of Variance ......

4. 566 Check

PLAT910 ALERT 3 C Missing \# of FCF Reflection(s) Below Theta(Min).

8 Note

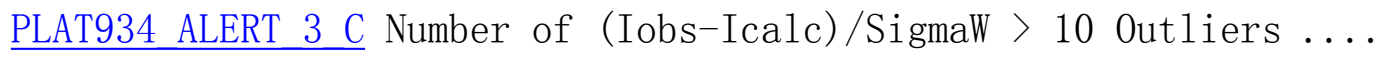

1 Check

\section{Alert level G}

PLAT199 ALERT 1 G Reported_cel1_measurement_temperature ..... (K)

293 Check

PLAT200 ALERT 1 G Reported diffrn ambient temperature ......(K)

293 Check

PLAT883 ALERT $1 \mathrm{G}$ No Info/Value for_atom_sites_solution_primary . Please Do !

PLAT978 ALERT 2 G Number C-C Bonds with Positive Residual Density.

7 Info

1 ALERT level $\mathrm{A}=$ Most likely a serious problem - resolve or explain

0 ALERT level $\mathrm{B}=\mathrm{A}$ potentially serious problem, consider carefully

4 ALERT level $\mathrm{C}=$ Check. Ensure it is not caused by an omission or oversight

4 ALERT level $\mathrm{G}=$ General information/check it is not something unexpected

4 ALERT type 1 CIF construction/syntax error, inconsistent or missing data 
2 ALERT type 2 Indicator that the structure model may be wrong or deficient

3 ALERT type 3 Indicator that the structure quality may be low

0 ALERT type 4 Improvement, methodology, query or suggestion

0 ALERT type 5 Informative message, check

It is advisable to attempt to resolve as many as possible of the alerts in all categories. Often the minor alerts point to easily fixed oversights, errors and omissions in your CIF or refinement strategy, so attention to these fine details can be worthwhile. In order to resolve some of the more serious problems it may be necessary to carry out additional measurements or structure refinements. However, the purpose of your study may justify the reported deviations and the more serious of these should normally be commented upon in the discussion or experimental section of a paper or in the "special_details" fields of the CIF. checkCIF was carefully designed to identify outliers and unusual parameters, but every test has its limitations and alerts that are not important in a particular case may appear. Conversely, the absence of alerts does not guarantee there are no aspects of the results needing attention. It is up to the individual to critically assess their own results and, if necessary, seek expert advice.

\section{Publication of your CIF in IUCr journals}

A basic structural check has been run on your CIF. These basic checks will be run on all CIFs submitted for publication in IUCr journals (Acta Crystallographica, Journal of Applied Crystallography, Journal of Synchrotron Radiation) ; however, if you intend to submit to Acta Crystallographica Section C or E or IUCrData, you should make sure that full publication checks are run on the final version of your CIF prior to submission.

\section{Publication of your CIF in other journals}


Please refer to the Notes for Authors of the relevant journal for any special instructions relating to CIF submission.

PLATON version of $03 / 05 / 2019$; check. def file version of $29 / 04 / 2019$

Datablock 20151125-hfp-1uo-1 - ellipsoid plot

Download CIF editor (publCIF) from the IUCr

Download CIF editor (enCIFer) from the CCDC

Test a new CIF entry 